\title{
Qualidade e transparência das pesquisas em saúde: Iniciativa EQUATOR aplicada à Fisioterapia
}

Helena Maria Silveira Fraga Maia (Helena Fraga-Maia) / ORCID: 0000-0002$2782-491$

Doutora - Professora Titular da Universidade do Estado da Bahia (UNEB) / Pesquisadora Associada do ELSA-Brasil

Lorena Rosa Santos de Almeida (Lorena R. S. Almeida) / ORCID: 0000-00034056-0208

Doutora - Fisioterapeuta do Hospital Geral Roberto Santos (HGRS/SESAB)

João de Deus Barreto Segundo (João de Deus Barreto Segundo) / ORCID: 00000002-4802-6045

Mestre - Editor Executivo do BAHIANA Journals (BAHIANA)

Objetivo do capítulo: Avaliar a qualidade e a transparência de relatos de pesquisa em saúde publicados em 2017 por fisioterapeutas brasileiros em revistas nacionais e internacionais de acordo com a Iniciativa EQUATOR e com a adesão a critérios de qualidade do International Committee of Medical Journals Editors - ICMJE.

Ao final do capítulo, espera-se que o leitor seja capaz de compreender os princípios e a relevância de descrever seus achados com transparência, integralidade e qualidade, assim como a necessidade de utilizar as diretrizes para relatos da Iniciativa EQUATOR de acordo com o desenho de estudo escolhido, tanto na elaboração do projeto, quanto na redação do relato de pesquisa em saúde.

- A translação do conhecimento depende fundamentalmente da transparência, integralidade e qualidade dos relatos.

- As diretrizes para relatos e seus respectivos checklists da Iniciativa EQUATOR são facilmente encontrados, mas não correntemente utilizados. 
- A transparência dos relatos varia de acordo com o QUALIS do periódico.

- A adesão às diretrizes para relatos pelas revistas é importante para ampliar o conhecimento e a utilização dessas ferramentas.

- As revistas que aderem à iniciativa EQUATOR e ICMJE têm, no geral, melhor desempenho de citação.

\section{Sobre a comunicação científica e a translação dos conhecimentos}

É inegável a contribuição das ciências para o avanço do conhecimento e dos cuidados à saúde. Todavia, não basta desenvolver estudos com excepcional rigor metodológico e máxima atenção aos princípios éticos. A ciência, para ser disseminada, precisa ser comunicada com uma redação precisa e clara, além de também ser comentada e verificada para ser aceita entre os pares ${ }^{1}$. Para tanto, quanto mais transparentes, completos e de boa qualidade forem os relatos de pesquisa em saúde, maior será a probabilidade da difusão dos novos conhecimentos gerados e sua incorporação na prática clínica para solucionar problemas individuais ou de coletividades ${ }^{2}$. Este processo, chamado de translação do conhecimento ${ }^{1-4}$, seria uma forma melhor de aproveitar todos os recursos aplicados por pesquisadores de forma ética com base em evidências científicas. Seria também uma forma de mensurar a qualidade das pesquisas, e muitos estudiosos têm investido para que esta forma impere e substitua a avaliação bibliométrica ${ }^{4}$. Todavia, esta última ainda tem sido uma das mais utilizadas e factíveis formas de avaliar a produção e disseminação do conhecimento. Por conta disto, é importante que, ao se analisarem os indicadores bibliométricos e cientométricos, o pesquisador não dependa de um único indicador, mas faça um desenho de estudo que cruze vários indicadores para uma análise final mais completa.

Facilmente pode-se comprovar que a pesquisa e a robustez das evidências em Fisioterapia têm avançado bastante, muito em função do expressivo aumento do número de doutores entre os profissionais da área ${ }^{5}$. Ainda assim, a atuação fisioterapêutica baseada em evidências apresenta grandes obstáculos, tais como uma alegada falta de tempo dos profissionais, de habilidade de leitura na língua inglesa, de incentivo dos empregadores em usar os resultados de 
pesquisas atuais na prática clínica, de treinamento formal em estratégias de buscas de literatura atual e relevante para a prática clínica e a dificuldade na obtenção de artigos na íntegra ${ }^{5-8}$. De fato, algumas destas barreiras poderiam ser eliminadas com uma boa formação profissional. Investir na qualificação do fisioterapeuta desde a fase mais precoce da graduação, estimulando o raciocínio científico, proporcionando conhecimentos para a captação e avaliação crítica da literatura e consequentemente impactando na habilidade de redigir cientificamente deve ser, portanto, uma obrigação dos docentes e das Instituições de Ensino Superior ${ }^{9}$. Tais práticas contribuem, não só para uma atuação baseada em evidências quanto para a produção científica mais qualificada, reduzindo a lacuna entre a pesquisa e a prática clínica. Reinders et al. ${ }^{10}$ observaram que alunos com experiência em pesquisas científicas publicam mais trabalhos após a graduação do que os que não tiveram essa prática. Porém, mesmo para aqueles que não foram treinados na escrita científica desde os primórdios da formação, várias diretrizes e checklists podem contribuir para a redação de projetos e de relatos de pesquisa em saúde que primem pela transparência, integralidade e qualidade.

A Iniciativa EQUATOR disponibiliza uma série de documentos, ou diretrizes, elaborados com o propósito de auxiliar autores para reportar adequadamente métodos e achados de pesquisas científicas ${ }^{11}$ desde março de 2006. As diretrizes e os checklists são facilmente localizados, encontram-se disponíveis em vários idiomas, inclusive o português, e já são adotados em normas de submissão de artigos por vários periódicos nacionais e internacionais que, adicionalmente, também adotam como obrigatória a observância aos postulados do Comitê Internacional de Editores de Periódicos Médicos - International Committee of Medical Journal Editors (ICMJE) que, desde o final dos anos 70, tem se preocupado com o conteúdo das publicações científicas. Porém, a despeito da facilidade de encontrar tais guias de redação, facilmente podemos observar que não são sempre seguidos, quer porque o corpo editorial de algumas revistas não os exigem, quer porque uma escrita mais completa, detalhada e clara também exige mais do pesquisador e requer mais experiência. Como a publicação de relatos de pesquisa tem sido uma moeda importante para obtenção de vantagens profissionais ${ }^{12}$, muitas vezes somam-se os desejos dos 
editores comerciais aos dos pesquisadores ainda em formação e assim, comunicações científicas são publicizadas sem os critérios que deveriam ser respeitados para favorecer o avanço dos conhecimentos, a Fisioterapia Baseada em Evidências, as avaliações mais consistentes e as intervenções apropriadas.

Para oportunizar o uso destes documentos e a observância de critérios muito caros à produção científica na área da Fisioterapia, avaliamos a qualidade e a transparência da produção recente de profissionais brasileiros.

\section{Avaliando a transparência e a qualidade dos relatos de pesquisa em saúde}

Uma amostra aleatória de estudos publicados em 2017 por fisioterapeutas brasileiros em revistas nacionais e internacionais foi selecionada nas bases de dados PubMed, ScienceDirect, Biblioteca Virtual em Saúde (BVS) e SciELO. Foram incluídos textos completos escritos em inglês ou português selecionados pelos desenhos dos estudos com os descritores "cross-sectional" OR "randomised trials" OR "systematic review". Foram ainda utilizados os descritores "Physiotherapy" OR "Physical Therapy" AND "Brazil" AND "2017". Para a seleção ainda foi necessário avaliar o Qualis dos periódicos em que foram publicados os estudos de modo a se obter, em igual número, artigos de revistas classificadas de $A 1$ a $B 1$ e de $B 2$ a B5. Os tipos de tratamentos fisioterapêuticos ou as subdisciplinas da Fisioterapia não foram considerados como critérios de inclusão.

Avaliamos a transparência dos relatos com a aplicação dos seguintes checklists da Iniciativa EQUATOR: o STROBE, o CONSORT e o PRISMA, para os desenhos transversais, ensaios clínicos e revisões sistemáticas, respectivamente. Estes desenhos de estudo foram escolhidos por representarem a maioria dos relatos de pesquisa realizados. A avaliação foi feita de modo cego pelas duas autoras fisioterapeutas e, em caso de não concordância, buscou-se o consenso. A assunção explícita aos propósitos da Iniciativa EQUATOR ou ao International Committee of Medical Journal Editors (ICMJE) nas normas de submissão dos periódicos foi pesquisada. 
O desempenho mensurado foi do ano de 2017 para garantir a maior atualidade possível aos dados e o período de coleta foi entre $1^{\circ}$ de março e 17 de abril de 2018. Os dados foram vistoriados e tratados antes de serem incluídos na tabela para exclusão de duplicatas. A qualidade das publicações foi avaliada de acordo com o QUALIS no quadriênio 2013-2016 da Plataforma Sucupira na área de avaliação Interdisciplinar e também por meio de indicadores bibliométricos como os Índices $\mathrm{h}, \mathrm{hl}$ Anual, AW, $\mathrm{g}$ e e.

O Índice $\mathbf{h}$ quantifica a produtividade e o impacto de cientistas ou revistas baseando-se nos seus artigos mais citados. Para tanto, corresponde ao número de artigos com citações maiores ou iguais a esse número, mostrando nuances que o Fator de Impacto não consegue alcançar. $O$ Índice g quantifica a produtividade científica individual (de autor e/ou título de revista) de acordo com o seu histórico de publicação. É, portanto, um indicador de contexto, pois é calculado sobre a distribuição de citações recebidas, criando assim uma média de citações que o Índice $\mathbf{h}$ não alcança. Ou seja, em um grupo de artigos listado em ordem decrescente de citações, o Índice $\mathbf{g}$ é o maior número único de modo que $\mathrm{o}$ artigo mais citado $\mathbf{g}$ recebeu pelo menos $\mathbf{g}^{2}$ citações. $\mathrm{O}$ Índice e é um balizador e complemento do Índice $\mathbf{h}$, que serve para melhor compreender grupos de pesquisadores ou revistas com Índices $\mathbf{h}$ similares, permitindo assim a comparação das subáreas da fisioterapia independentemente da possibilidade de que uma publique mais frequentemente do que outra. $\mathrm{O} \mathrm{hl}$ Anual permite comparar fontes em diferentes estágios de desenvolvimento, permitindo a comparação de desempenho de revistas que publicam há mais tempo com revistas que publicam há menos. É calculado ao se dividir a Norma hl pelo tempo de publicação desde a primeira publicação. O Índice AW é a raiz quadrada da Taxa de Citação AW, que normaliza as citações no tempo considerando a idade de cada artigo individualmente. Ou seja, o número de citações de cada artigo é dividido pela idade do artigo. O Índice AW permite comparação com o Índice $\mathbf{h}$.

Optamos pela recuperação dos indicadores de desempenho cientométrico utilizando o título completo do periódico. Os indicadores foram calculados no software gratuito Harzing's Publish or Perish, versão 6.28.6197.6663. No 
software, a base de dados utilizada para todas as revistas foi o Microsoft Academic e um só autor realizou a coleta para minimizar a ocorrência de viés.

Quando a busca pelo título completo do periódico não devolveu nenhuma atividade de citação, empregamos a abreviação oficial do título da revista conforme descrito no catálogo da National Library of Medicine (NLM), do National Center for Biotechnology Information estadounidense. Os títulos foram confirmados no catálogo da NLM através do International Standard Serial Number (ISSN). A NLM cataloga periódicos independentemente de sua indexação no MEDLINE/PubMed.

O desempenho mensurado foi de 2017 ao período de coleta - que ocorreu entre $1^{\circ}$ de março e 17 de abril de 2018 - para garantir a maior atualidade possível aos dados. Os dados foram vistoriados e tratados antes de serem incluídos na tabela para exclusão de duplicatas (artigos publicados na revista e em repositórios institucionais) e de dados de revistas homônimas misturados nos resultados da busca. Para este fim foi considerada a aba Publication em detrimento à aba Publisher no software. Revistas que em observação a estes parâmetros de busca não obtiveram citações tiveram seus indicadores zerados.

\section{O que selecionamos para avaliar a transparência e a qualidade dos relatos de pesquisa em saúde e o que encontramos}

Foram selecionados 36 textos completos, sendo 35 disponíveis para download gratuito. Foram incluídos 12 relatos de cada um dos desenhos de estudo escolhidos para avaliação dos checklists da Iniciativa EQUATOR e, dentre estes, seis provenientes de revistas classificadas de A1 a B1 e outros seis de B2 a B5 (QUALIS/CAPES). Deste modo, foram elegidos intencionalmente, além de relatos publicados em periódicos de boa qualidade, artigos de revistas não indexadas no MEDLINE e sem uma clara política editorial obrigando a adesão aos checklists voltados para garantir transparência e qualidade aos estudos. Apenas um artigo não foi disponibilizado online, tendo sido adquirido por solicitação ao Conselho Regional de Fisioterapia e Terapia Ocupacional (CREFITO 7), detentor de assinatura do periódico. 
$\mathrm{Na}$ Tabela 1, dispomos os itens do checklist STROBE para estudos seccionais publicados por fisioterapeutas brasileiros em 2017 e seus respectivos títulos abreviados, estado em que foi realizada a pesquisa, e periódico de acordo com o QUALIS no quadriênio 2013-2016 (Plataforma Sucupira). As principais discrepâncias, poucas, mas existentes, entre os relatos, de acordo com os estratos do QUALIS, recaíram sobre a ausência de definição clara das variáveis ou seja, de todos os desfechos, exposições, preditores, confundidores em potencial e modificadores de efeito, a falta da indicação da fonte dos dados e os detalhes dos métodos utilizados na avaliação ou mensuração, a inexistência de dados sobre das medidas adotadas para evitar potenciais fontes de viés, ausência de informações sobre como foram tratadas as variáveis quantitativas na análise e as categorizações que foram adotadas, e, finalmente, a ausência de uma discussão acerca da generalização, ou validade externa, dos resultados. Pode-se observar uma maior transparência entre os relatos de pesquisa que foram publicados no estrato A1 a B1.

$\mathrm{Na}$ Tabela 2, disponibilizamos as informações obtidas com a aplicação do checklist CONSORT para ensaios clínicos randomizados e seus respectivos títulos abreviados, estado em que foi realizada a pesquisa e periódico de acordo com o QUALIS no quadriênio 2013-2016 (Plataforma Sucupira). Sobre o registro obrigatório dos ensaios clínicos observou-se que, no estrato A1 a B1 apenas em uma revista (brasileira) não constava a informação sobre sua adequada realização. Já no estrato B2 a B5, todos periódicos nacionais, verificou-se que 3 dos 6 artigos também não apresentaram o referido registro. De modo geral, foi possível identificar, para os dois grupos do QUALIS, ausência da transparência na determinação do tamanho amostral, assim como a inexistência de uma discussão sobre a generalização, com informação sobre a aplicabilidade dos achados do estudo clínico. Porém, observou-se superioridade na escrita dos artigos do bloco A1 a B1 quanto à descrição das intervenções de cada grupo com detalhes suficientes para permitir a replicação, do método utilizado para geração de sequência randomizada de alocação e do mecanismo para implementá-la, dos tipos de randomização, das informações sobre quem gerou a sequência de alocação randomizada, sobre quem inscreveu os participantes e 
quem atribuiu as intervenções aos participantes, quanto ao fornecimento de informações sobre o cegamento após as intervenções terem sido atribuídas, quanto à apresentação do fluxograma e dos dados de base demográficos e características clínicas dos grupos, além da descrição acerca de danos ou efeitos indesejados em cada grupo.

Os itens do checklist PRISMA para revisões sistemáticas da literatura e seus respectivos títulos abreviados, estado em que foi realizada a pesquisa, e periódico de acordo com o QUALIS no quadriênio 2013-2016 (Plataforma Sucupira) apresentamos na Tabela 3. De modo geral, chama a atenção a ausência de informações sobre a existência de um protocolo e do registro das revisões sistemáticas. A listagem e definição das variáveis obtidas dos dados, o risco de viés em cada estudo, o risco de viés entre os estudos, as medidas de sumarização e a síntese dos resultados no item Métodos, assim como os tópicos do item Resultados, e as limitações e conclusões no item Discussão foram os pontos que mais divergiram entre as publicações de acordo com o estrato do QUALIS. Ou seja, foi identificada uma grande diferença em termos de transparência entre os relatos, com predomínio claro de qualidade entre os publicados no estrato $A 1$ a B1.

Já na Tabela 4, exibimos os periódicos dos artigos selecionados, a qualidade dos mesmos avaliada de acordo com o QUALIS CAPES nas duas categorias, os indicadores bibliométricos e a assunção explicita aos propósitos da Iniciativa EQUATOR ou do International Committee of Medical Journal Editors nas normas de publicação. Metade dos 16 periódicos incluídos no estrato A1 a B1 eram internacionais, enquanto todos inseridos no estrato B2 a B5 eram nacionais. De modo geral, observa-se que as revistas que aderem às iniciativas EQUATOR e ICMJE têm, no geral, melhor desempenho de citação no ano imediatamente posterior à sua publicação.

Nas revistas em que foram publicados os artigos selecionados, especificamente para os ensaios clínicos randomizados, as normas para os autores submeterem suas publicações são claras e cinco (83\%) das 6 listadas no estrato de A1 a B1 exigem o registro do ensaio clinico, sendo a única que não exige uma revista 
nacional. Já no estrato B2 a B5, nenhum dos seis periódicos especificam esta exigência.

\section{Análise dos relatos de pesquisa em saúde selecionados que foram publicados em 2017 por fisioterapeutas brasileiros}

Entre as 15 revistas no estrato B2 a B5, 12 (80\%) declararam assunção aos propósitos do ICMJE, mas apenas duas à Iniciativa EQUATOR e ao ICMJE simultaneamente. Entre estas, apenas a Revista Pesquisa em Fisioterapia (ISSN 2238-2704) obteve atividade de citação no intervalo analisado de modo a obter variação nos índices observados. Embora não se possa concluir definitivamente que apenas a assunção ao ICMJE e à Iniciativa EQUATOR resultem em melhor desempenho cientométrico, há evidência neste segmento da amostra que a não assunção aos princípios de transparência está correlacionado com baixa ou nenhuma atividade de citação no ano posterior à publicação de artigos inéditos.

Poder-se-ia especular que um ano é pouco tempo para obtenção de citações e que a maioria dos artigos começa a ser citado após o segundo ano de publicação, por conta inclusive do tempo necessário à revisão por pares, estimado em 12 a 18 meses nas revistas mais bem reputadas. Porém, a atividade de citação expressiva no recorte amostral referente ao estrato $A 1$ a B1 contesta esta possibilidade. Fica evidente ao se observar este estrato que artigos podem ser citados no ano imediatamente posterior à sua publicação, fortalecendo a hipótese de que a não assunção às iniciativas EQUATOR e do ICMJE prejudica a reputação da revista e a torna menos confiável, podendo zerar as suas citações no ano imediatamente posterior à sua publicação.

Todos os periódicos do estrato A1 a B1 possuem desempenho cientométrico significativo. Nesta secção da amostra, há evidência de que a estratificação QUALIS está correlacionada com atividade de citação de regular a expressiva. Entretanto, a estratificação QUALIS não retrata necessariamente assunção explícita às iniciativas de fortalecimento dos princípios científicos: as duas revistas com melhor desempenho na amostra como um todo e nesta secção da 
amostra não aderem aos checklists EQUATOR embora adiram às orientações do ICMJE - European Journal of Obstetrics \& Gynecology and Reproductive Biology (0301-2115) e a Revista Fisioterapia em Movimento (1980-5918). Vale ressaltar, entretanto que essas duas revistas representam apenas $12 \%$ do estrato A1 - B1 da amostra.

Ainda sobre o estrato A1 a B1, aproximadamente $29 \%$ das revistas não aderem totalmente às iniciativas. Ou seja, normalmente, adesão a iniciativas de transparência e padronização de relatos de pesquisa aparecem em conjunto na amostra com maior atividade de citação, sugerindo uma relação causal: se há adesão, logo há citação. Contudo, os dados sugerem que pode haver outros fatores operando na reputação e atratividade das revistas European Gynecology e Fisioterapia em Movimento que as coloquem no topo como as de maior atividade de citação: European Journal of Obstetrics \& Gynecology e Autonomic Neuroscience são publicadas pela Elsevier, editora comercial com estratégias de comunicação e marketing científico diferenciados, além de capital para implementar essas estratégias. A Journal of Physiotherapy, também publicada pela Elsevier, é a revista da Australian Physiotherapy Association, então detém também capital simbólico no campo posto que é a revista oficial da entidade de classe daquele país. Apesar de acumular esses dois predicados de prestígio à sua reputação, a Journal of Physiotherapy não está entre as revistas de maior atividade de citação deste segmento da amostra, o que pode sinalizar que apenas estar em uma editora comercial e vinculado a entidade de classe não resulta necessariamente em maior atividade de citação.

A Revista Fisioterapia em Movimento, por sua vez, recebe apoio financeiro apenas de fundações: a mantenedora da Pontifícia Universidade Católica do Paraná e a de apoio à pesquisa do estado, a Fundação Araucária. Também em A1-B1, Autonomic Neuroscience: Basic and Clinical (1566-0702), que não adere a nenhuma das iniciativas, e a Revista Saúde \& Transformação Social (21787085), que adere apenas à Iniciativa EQUATOR, estão respectivamente nas posições última e antepenúltima, considerando o ranking a partir do índice e para domínios distintos do conhecimento. A Revista Saúde \& Transformação Social obteve zero em todos os índices, reforçando que apesar de estar no 
estrato $A 1-B 1$, a recusa às iniciativas de transparência e padronização de relatos recorrem nos resultados deste estudo associadas a nenhum desempenho de citação no primeiro ano logo após a publicação. Autonomic Neuroscience recusa ambas as iniciativas e apesar disto possui uma taxa de citação normalizada no tempo expressiva.

Dos 12 ensaios clínicos selecionados $8(67 \%)$ foram registrados. Entre os publicados no estrato $A 1$ a B1, $83 \%$, e no estrato B2 a B5, 50\%. Se considerarmos que o registro foi proposto no final dos anos 90 , é possível afirmar que não se trata de uma novidade no meio científico. Em 2016, Chan \& Heinemann ${ }^{11}$ publicaram o Editorial do The American Journal of Occupational Therapy intitulado "Clinical Trial Registration: The Time Has Come". Neles, os pesquisadores chamam a atenção para o fato de que este registro passou a ser obrigatório desde $1^{\circ}$ de janeiro de 2016 para as revistas internacionais relacionadas com reabilitação, sendo que um consórcio formado por editores das revistas de Fisioterapia (International Society of Physiotherapy Journal Editors) informou aos leitores, em janeiro de 2013, que transformou o registro de opcional para obrigatório no referido ano. Podemos considerar que os ensaios clínicos publicados por fisioterapeutas brasileiros em 2017 tenham começado em anos anteriores e por isso não tenham sido registrados, mas também é preciso informar que é possível o registro mesmo para os estudos já em andamento. Em relação às revisões sistemáticas, apenas duas (17\%) foram registradas. Ambas pertenciam ao estrato $\mathrm{A} 1$ a $\mathrm{B} 1$, correspondendo a $33 \%$ dos relatos nesta categoria. Apesar de até o momento não ser obrigatório, o registro de uma revisão sistemática é fortemente recomendado ${ }^{13}$. O registro tanto de ensaios clínicos quanto de revisões sistemáticas pode contribuir para evitar publicações seletivas, prevenir desnecessária duplicação de estudos em andamento, e informar pacientes e o público em geral sobre o que está sendo alvo de investigações científicas, dentre outros benefícios ${ }^{13,14}$.

Dentre os três desenhos de estudo avaliados, os seccionais foram os que apresentaram maior cumprimento de apresentação dos itens do checklist da Iniciativa EQUATOR e, consequentemente, menor discrepância em relação à transparência dos relatos entre os estratos A1 a B1 e B2 a B5. Isso pode ser 
atribuído à maior simplicidade do referido desenho de estudo e facilidade para sua elaboração e execução quando comparado aos ensaios clínicos randomizados e às revisões sistemáticas. Um ponto em comum aos três desenhos de estudo, não restrito aos relatos publicados em periódicos do estrato B2 a B5, mas principalmente neles, foi a ausência de informações sobre a generalização dos achados da pesquisa. A generalização depende de uma série de elementos que devem ser contemplados nos relatos, como critérios de elegibilidade, informações sobre quando e onde os dados foram coletados e tamanho amostral ${ }^{14,15}$. Dessa forma, o julgamento sobre a generalização de um estudo não só está diretamente relacionado com a transparência com que os itens determinados pelas diretrizes para relatos são apresentados, como também pode impactar na qualidade da pesquisa.

\section{Concluindo nossas observações sobre o relatos selecionados}

Em geral, observamos que a qualidade e a transparência dos relatos de pesquisa em saúde, publicados por fisioterapeutas brasileiros no recorte de tempo estudado (2017), variaram conforme o estrato QUALIS no quadriênio 2013-2016 (Plataforma Sucupira). Aqueles pertencentes ao estrato A1 a B1 apresentaram maior adesão aos itens das diretrizes para relatos da Iniciativa EQUATOR, gerando uma maior transparência do artigo científico e contribuindo para melhores indicadores bibliométricos dos periódicos nos quais os relatos foram publicados.

É fundamental que os autores tenham conhecimento das diretrizes para relatos de pesquisa em saúde desde o momento de elaboração do projeto de pesquisa, para evitar que itens essenciais deixem de ser contemplados, e que utilizem os checklists para guiar a redação do artigo científico. Por outro lado, é essencial à adesão às diretrizes para relatos por parte dos periódicos não só para difundir o conhecimento como também para aumentar a utilização de tais ferramentas, o que está atrelado a um melhor desempenho de citação. Por fim, vale salientar que a transparência dos relatos, isoladamente, não garante a qualidade dos mesmos. Todavia, cumprir todos os tópicos do checklist referente ao desenho de estudo adotado pelo pesquisador requer dele conhecimento do método 
científico e experiência em pesquisa, que pressupõem a realização de uma produção científica com maior qualidade. Desta forma, a translação do conhecimento seria mais facilmente alcançada e contribuiria para a prática baseada em evidências na área da Fisioterapia. 
Tabela 1. Checklist STROBE para estudos seccionais publicados por fisioterapeutas brasileiros em 2017 e seus respectivos títulos abreviados, estado em que foi realizada a pesquisa, e periódico de acordo com o QUALIS no quadriênio 2013-2016 (Plataforma Sucupira), 2018.

\begin{tabular}{|c|c|c|c|c|c|c|c|c|c|c|c|c|}
\hline \multirow{3}{*}{$\begin{array}{l}\text { QUALIS - Plataforma Sucupira } \\
\text { Título abreviado } \\
\text { Estado do Brasil em que foi } \\
\text { realizada a pesquisa }\end{array}$} & \multicolumn{6}{|c|}{ A1 a B1 } & \multicolumn{6}{|c|}{ B2 a B5 } \\
\hline & 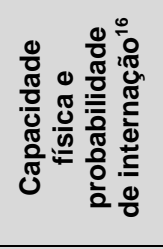 & 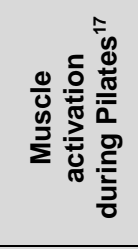 & 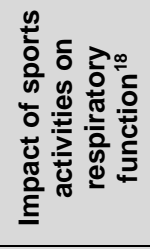 & 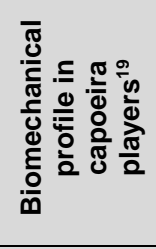 & 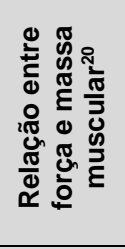 & 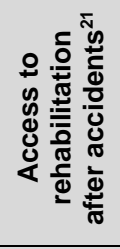 & 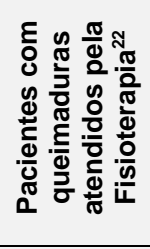 & 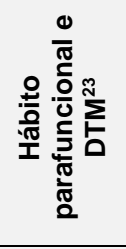 & 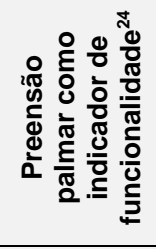 & 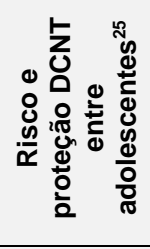 & 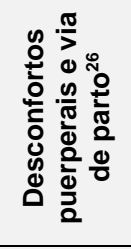 & 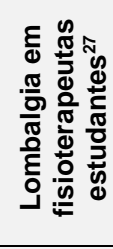 \\
\hline & RS & SP & SC & CE & $\mathbf{R N}$ & RN & GO & MA & BA & MA & SP & GO \\
\hline Periódico & $\begin{array}{l}\frac{1}{ \pm} \\
\frac{5}{0} \\
\frac{0}{0} \\
\frac{0}{4}\end{array}$ & 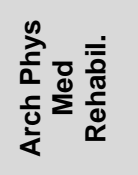 & $\frac{\grave{d}}{\frac{0}{n}} \frac{0}{2}$ & 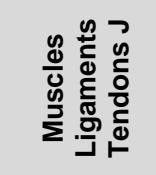 & 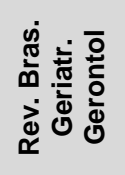 & 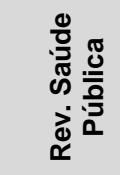 & 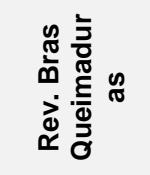 & 占 & 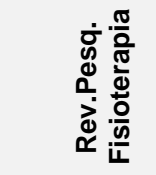 & 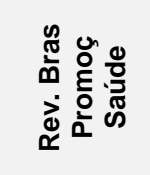 & 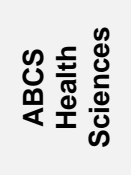 & 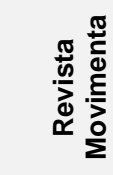 \\
\hline
\end{tabular}

Strengthening the Reporting of Observational Studies in Epidemiology (STROBE) para estudos seccionais

\section{Título e resumo}

Introdução

Contexto/justificativa

Métodos Objetivos

Desenho do estudo
Contexto
Participantes
Variáveis: definição clara
Fontes de dados/
mensuração
Viés
Tamanho amostral
Variáveis quantitativas
Métodos estatísticos
Resultados
Participantes
Dados descritivos

$\begin{array}{ll}x & x \\ x & x \\ x & x \\ x & x \\ x & x \\ x & x \\ x & x \\ x & x \\ x & x \\ x & x \\ x & x \\ x & x \\ x & x \\ x & x\end{array}$

$\begin{array}{llll}\mathrm{X} & \mathrm{X} & \mathrm{X} & \mathrm{X} \\ \mathrm{X} & \mathrm{X} & \mathrm{X} & \mathrm{X} \\ \mathrm{X} & \mathrm{X} & \mathrm{X} & \mathrm{X} \\ \mathrm{X} & \mathrm{X} & \mathrm{X} & \mathrm{X} \\ - & - & \mathrm{X} & \mathrm{X} \\ \mathrm{X} & \mathrm{X} & \mathrm{X} & \mathrm{X} \\ \mathrm{X} & \mathrm{X} & \mathrm{X} & \mathrm{X} \\ \mathrm{X} & \mathrm{X} & \mathrm{X} & \mathrm{X} \\ \mathrm{X} & \mathrm{X} & \mathrm{X} & \mathrm{X} \\ \mathrm{X} & - & \mathrm{X} & \mathrm{X} \\ \mathrm{X} & \mathrm{X} & \mathrm{X} & \mathrm{X} \\ \mathrm{X} & \mathrm{X} & \mathrm{X} & \mathrm{X} \\ \mathrm{X} & \mathrm{X} & \mathrm{X} & \mathrm{X} \\ \mathrm{X} & \mathrm{X} & \mathrm{X} & \mathrm{X}\end{array}$

\begin{tabular}{l}
$x$ \\
$x$ \\
$x$ \\
$x$ \\
$x$ \\
$x$ \\
- \\
- \\
- \\
$x$ \\
\hline$x$ \\
$x$ \\
$x$ \\
$x$
\end{tabular}

$X$

$\mathrm{X}$

Dados descritivos 
Desfecho Outras análises

Discussão

Resultados principais

Limitações

Interpretação

Generalização

Outras informações

Financiamento 
Tabela 2. Checklist CONSORT para ensaios clínicos randomizados publicados em 2017 por fisioterapeutas brasileiros e seus respectivos títulos abreviados, estado em que foi realizada a pesquisa e periódico de acordo com o QUALIS no quadriênio 2013-2016 (Plataforma Sucupira), 2018.

\begin{tabular}{|c|c|c|c|c|c|c|c|c|c|c|c|c|}
\hline \multirow[t]{2}{*}{ QUALIS - Plataforma Sucupira } & \multicolumn{6}{|c|}{ A1 a B1 } & \multicolumn{6}{|c|}{ B2 a B5 } \\
\hline & 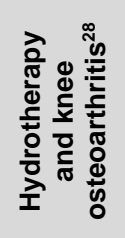 & 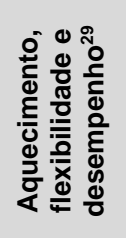 & 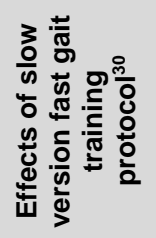 & 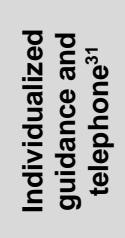 & 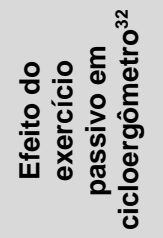 & 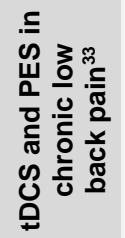 & 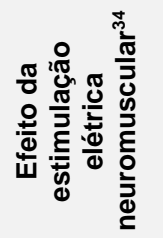 & 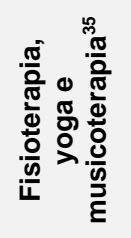 & 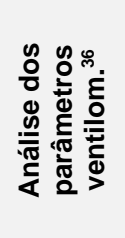 & 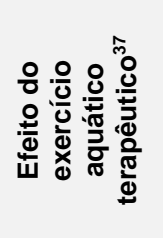 & 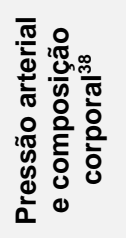 & 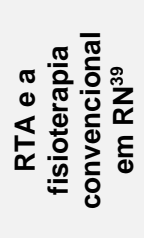 \\
\hline $\begin{array}{l}\text { Estado do Brasil em que foi } \\
\text { realizada a pesquisa }\end{array}$ & MG & RN & SP & PE & RS & SP & RS & sc & PA & sc & SP & $\mathbf{R J}$ \\
\hline Periódico & 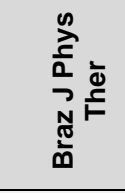 & 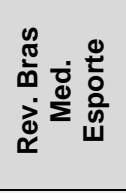 & 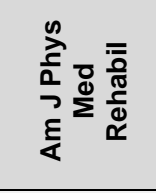 & $\frac{\bar{d}}{\frac{1}{0}} \frac{0}{0}$ & 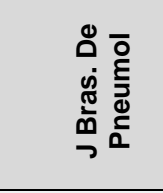 & 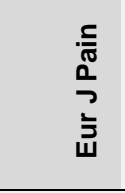 & 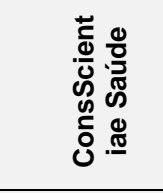 & 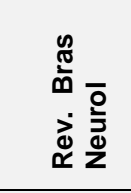 & 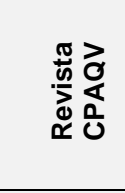 & 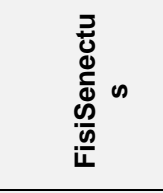 & 흉 & 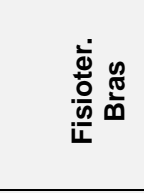 \\
\hline \multicolumn{13}{|c|}{ Check-list Consolidated Standards of Reporting Trials (CONSORT) para estudos clínicos randomizados } \\
\hline Título & $\mathrm{x}$ & $\mathrm{x}$ & $x$ & - & $x$ & $\mathrm{x}$ & $x$ & - & $x$ & $\mathrm{x}$ & $\mathrm{x}$ & $\mathrm{x}$ \\
\hline $\begin{array}{l}\text { Resumo } \\
\text { Introdução }\end{array}$ & $x$ & $x$ & $x$ & $x$ & $x$ & $x$ & $x$ & $x$ & $x$ & $x$ & $x$ & $x$ \\
\hline Fundamentação científica & $x$ & $x$ & $x$ & $x$ & $x$ & $x$ & $x$ & $x$ & $x$ & $x$ & $x$ & $x$ \\
\hline Objetivos específicos & $\mathrm{x}$ & $\mathrm{x}$ & $\mathrm{x}$ & $\mathrm{x}$ & $\mathrm{x}$ & $\mathrm{x}$ & $\mathrm{X}$ & $x$ & $\mathrm{x}$ & $x$ & $\mathrm{x}$ & $x$ \\
\hline 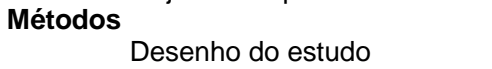 & & & & & & & & & & & & \\
\hline Descrição & $\mathrm{x}$ & $\mathrm{x}$ & $\mathrm{x}$ & $\mathrm{x}$ & $x$ & $\mathrm{x}$ & $\mathrm{x}$ & $\mathrm{x}$ & $\mathrm{x}$ & $\mathrm{x}$ & - & $\mathrm{x}$ \\
\hline Alteração nos métodos & - & - & - & - & - & - & - & - & - & - & - & - \\
\hline Participantes & & & & & & & & & & & & \\
\hline Elegibilidade & $\mathrm{x}$ & $\mathrm{x}$ & $\mathrm{x}$ & $\mathrm{x}$ & $\mathrm{x}$ & $\mathrm{x}$ & $x$ & $\mathrm{x}$ & $\mathrm{x}$ & $\mathrm{x}$ & $\mathrm{x}$ & $\mathrm{x}$ \\
\hline Informações e locais & $x$ & $x$ & $x$ & $x$ & $x$ & $x$ & $x$ & $x$ & $x$ & $x$ & - & $x$ \\
\hline $\begin{array}{l}\text { Intervenções } \\
\text { Desfechos }\end{array}$ & $x$ & $x$ & $x$ & $x$ & $x$ & $x$ & $x$ & $x$ & - & - & $x$ & - \\
\hline Primários e secundários & $x$ & $\mathrm{X}$ & $\mathrm{x}$ & $\mathrm{X}$ & $\mathrm{x}$ & $\mathrm{X}$ & $\mathrm{x}$ & $x$ & $\mathrm{x}$ & - & $\mathrm{x}$ & $x$ \\
\hline $\begin{array}{l}\text { Alterações após início } \\
\text { Tamanho amostral }\end{array}$ & $x$ & - & - & - & - & $\mathrm{X}$ & - & - & - & - & - & - \\
\hline Determinação & $x$ & - & - & $x$ & - & $x$ & $x$ & - & $x$ & - & - & $x$ \\
\hline
\end{tabular}


Diretrizes de

encerramento

Randomização

Geração

Tipo

Alocação

Implementação

Cegamento

Quem e como

Semelhança das

intervenções

Métodos estatísticos

Métodos

Resultados

Analses adicionais

$$
\text { Fluxograma }
$$

Perdas e exclusões

Recrutamento

Datas e períodos

Estudo finalizado ou

Dados de base
Números analisado

Desfechos e estimativas

grupo Resultados de cada

Desfechos binários Análises auxiliares

\section{Discussão}

\section{Limitações \\ Generalização \\ Interpretação \\ Outras informações}

Apresenta o Registro

Informa o Protocolo

Fomento

Documentos adicionais disponíveis

para download, tais como

protocolos, imagens, vídeos, etc.

$\begin{array}{llllll}x & x & x & x & x & x \\ x & x & x & x & x & x \\ x & x & x & x & x & x \\ - & x & - & - & x & x \\ x & x & x & - & x & x \\ - & - & - & - & - & -\end{array}$

$x$
$x$
$x$
$x$
$x$
$x$

$\begin{array}{ll}x & x \\ x & x\end{array}$

$\mathrm{x}$


Tabela 3. Checklist PRISMA para revisões sistemáticas da literatura de artigos publicados por fisioterapeutas brasileiros em 2017 e seus respectivos títulos abreviados, estado em que foi realizada a pesquisa, e periódico de acordo com o QUALIS no quadriênio 2013-2016 (Plataforma Sucupira), 2018.

\begin{tabular}{|c|c|c|c|c|c|c|c|c|c|c|c|c|}
\hline \multirow[t]{2}{*}{ QUALIS - Plataforma Sucupira } & \multicolumn{6}{|c|}{ A1 a B1 } & \multicolumn{6}{|c|}{ B2 a B5 } \\
\hline & 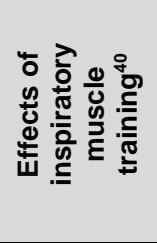 & 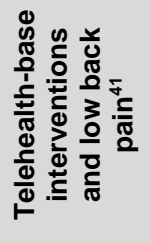 & 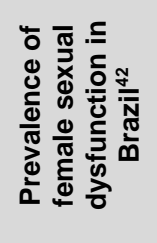 & 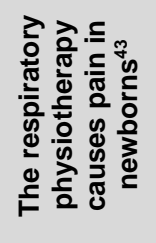 & 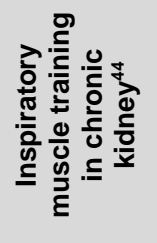 & 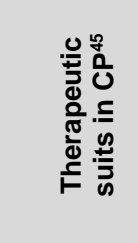 & 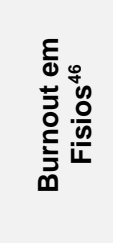 & 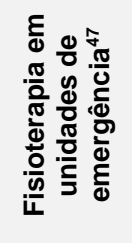 & 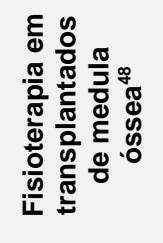 & 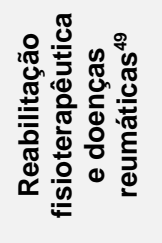 & 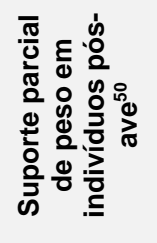 & 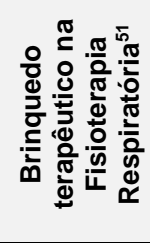 \\
\hline $\begin{array}{l}\text { Estado do Brasil em que foi } \\
\text { realizada a pesquisa }\end{array}$ & SP & SP/BA & SC & MG & PE & ES/MG & BA & BA & CE & RS & $\begin{array}{c}\text { SP/SE/ } \\
\text { MG }\end{array}$ & SC \\
\hline \multirow[t]{2}{*}{ Periódico } & 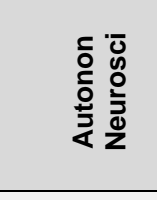 & $\begin{array}{l}\frac{0}{0} \\
\text { के }\end{array}$ & 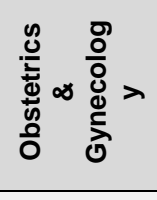 & $\frac{\frac{1}{ \pm}}{\frac{0}{0}} \frac{0}{i \frac{0}{L}}$ & 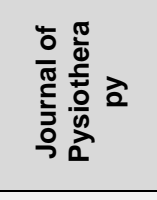 & 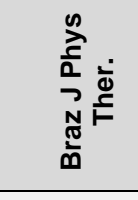 & 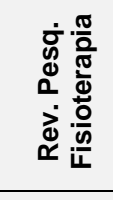 & 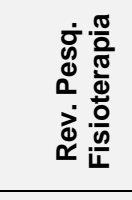 & 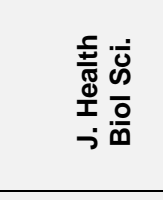 & 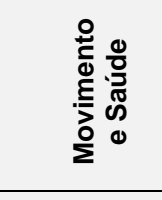 & 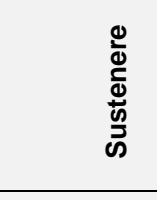 & 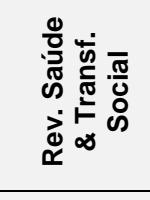 \\
\hline & \multicolumn{11}{|c|}{ Preferred Reporting Items for Systematic Reviews and Meta-Analyses (PRISMA) para Revisões Sistemáticas } & \\
\hline Título & $\mathrm{x}$ & $\mathrm{x}$ & $\mathrm{x}$ & $\mathrm{x}$ & $x$ & $\mathrm{x}$ & $\mathrm{x}$ & $\mathrm{x}$ & $\mathrm{x}$ & $\mathrm{x}$ & $\mathrm{x}$ & $\mathrm{x}$ \\
\hline $\begin{array}{l}\text { Resumo } \\
\text { Introdução }\end{array}$ & $\mathrm{x}$ & - & - & - & $x$ & $x$ & - & - & - & - & - & - \\
\hline Racional & $x$ & $x$ & $x$ & $x$ & $x$ & $x$ & $x$ & $x$ & $x$ & $\mathrm{x}$ & $\mathrm{x}$ & $x$ \\
\hline Métodos Objetivos & $\mathrm{x}$ & $x$ & $\mathrm{x}$ & $x$ & $x$ & $x$ & $\mathrm{x}$ & - & $x$ & - & - & $\mathrm{x}$ \\
\hline Protocolo e registro & - & $x$ & - & - & $x$ & - & - & - & - & _- & - & - \\
\hline Critérios de elegibilidade & $x$ & $\hat{x}$ & $x$ & $x$ & $\hat{x}$ & $x$ & $x$ & $x$ & $x$ & $x$ & $x$ & $x$ \\
\hline Fontes de informação & $x$ & $x$ & $x$ & $x$ & $x$ & $x$ & $\mathrm{x}$ & $x$ & $x$ & $\mathrm{x}$ & $\mathrm{x}$ & $x$ \\
\hline Busca & $x$ & $\mathrm{x}$ & $x$ & - & $x$ & $\mathrm{x}$ & $\mathrm{x}$ & $\mathrm{x}$ & $x$ & $x$ & - & $\mathrm{x}$ \\
\hline Seleção de estudos & $x$ & $x$ & $x$ & $x$ & $x$ & $x$ & $x$ & $x$ & - & $x$ & $x$ & $x$ \\
\hline $\begin{array}{l}\text { Processo de coleta de } \\
\text { dados }\end{array}$ & $\mathrm{x}$ & $x$ & $\mathrm{x}$ & $x$ & $\mathrm{x}$ & $\mathrm{x}$ & $\mathrm{x}$ & $\mathrm{x}$ & - & $x$ & - & $\mathrm{x}$ \\
\hline $\begin{array}{l}\text { Lista dos dados } \\
\text { Risco de viés em cada }\end{array}$ & $x$ & $x$ & - & - & $x$ & $x$ & - & & - & $x$ & - & - \\
\hline estudo & $\mathrm{x}$ & $x$ & $\mathrm{x}$ & $\mathrm{x}$ & $x$ & $\mathrm{x}$ & - & $\mathrm{x}$ & - & - & - & - \\
\hline Medidas de sumarização & $\mathrm{x}$ & $\mathrm{x}$ & - & - & $\mathrm{x}$ & - & - & - & - & - & - & - \\
\hline Síntese dos resultados & - & $x$ & - & - & $x$ & - & - & - & - & - & - & - \\
\hline
\end{tabular}




\begin{tabular}{|c|c|c|c|c|c|c|c|c|c|c|c|c|}
\hline \multirow{3}{*}{$\begin{array}{l}\text { Risco de viés entre os } \\
\text { estudos } \\
\text { Análises adicionais }\end{array}$} & & & & & & & & & & & & \\
\hline & - & $\mathrm{x}$ & - & - & $\mathrm{X}$ & - & - & - & - & - & - & - \\
\hline & - & - & - & - & - & - & - & - & - & - & - & - \\
\hline \multicolumn{13}{|l|}{ Resultados } \\
\hline $\begin{array}{l}\text { Seleção dos estudos } \\
\text { Características dos }\end{array}$ & $x$ & $\mathrm{x}$ & $\mathrm{x}$ & $\mathrm{x}$ & $\mathrm{x}$ & $\mathrm{x}$ & $\mathrm{x}$ & $\mathrm{x}$ & - & $\mathrm{x}$ & - & $\mathrm{x}$ \\
\hline $\begin{array}{l}\text { estudos } \\
\text { Risco de viés em cada }\end{array}$ & $x$ & $x$ & $x$ & $x$ & $x$ & $x$ & $x$ & - & - & - & - & - \\
\hline $\begin{array}{l}\text { estudo } \\
\text { Resultados dos estudos }\end{array}$ & - & $\mathrm{x}$ & $\mathrm{x}$ & - & $\mathrm{x}$ & $\mathrm{x}$ & - & - & - & - & - & - \\
\hline individuais & $\mathrm{x}$ & $\mathrm{x}$ & $\mathrm{x}$ & $x$ & $\mathrm{x}$ & $\mathrm{x}$ & $\mathrm{x}$ & - & - & - & - & - \\
\hline $\begin{array}{l}\text { Síntese dos resultados } \\
\text { Risco de viés entre }\end{array}$ & $\mathrm{x}$ & $\mathrm{x}$ & $\mathrm{x}$ & $\mathrm{x}$ & $\mathrm{x}$ & $\mathrm{x}$ & $\mathrm{x}$ & - & - & - & - & - \\
\hline estudos & - & $\mathrm{x}$ & - & - & $\mathrm{x}$ & - & - & - & - & - & - & - \\
\hline Análises adicionais & - & - & - & - & - & - & - & - & - & - & - & - \\
\hline \multicolumn{13}{|l|}{ Discussão } \\
\hline Sumário da evidência & $\mathrm{x}$ & $\mathrm{x}$ & $\mathrm{x}$ & $\mathrm{X}$ & $\mathrm{x}$ & $\mathrm{X}$ & $\mathrm{x}$ & - & - & - & - & - \\
\hline Limitações & $x$ & $\mathrm{x}$ & $x$ & $x$ & $\mathrm{x}$ & $\mathrm{x}$ & $\mathrm{x}$ & - & - & $\mathrm{x}$ & - & - \\
\hline Conclusões & $x$ & $\mathrm{x}$ & $x$ & $x$ & $\mathrm{x}$ & $x$ & $\mathrm{x}$ & - & $x$ & - & - & - \\
\hline Financiamento & - & - & $x$ & - & $\mathrm{x}$ & $x$ & - & - & - & - & - & - \\
\hline
\end{tabular}


Tabela 4. Periódicos acessados para seleção de artigos, qualidade dos periódicos, cientometria e assunção explicita aos propósitos da Iniciativa EQUATOR ou referência de adequação ao International Committee of Medical Journal Editors nas normas de publicação, de acordo com o QUALIS, 2018

\begin{tabular}{|c|c|c|c|c|c|c|c|c|c|c|}
\hline PERIÓDICOS (ISSN eletrônico) & QUALIS & Índice $\mathrm{h}$ & $\begin{array}{l}\text { Índice hl } \\
\text { Annual }\end{array}$ & $\begin{array}{c}\text { Citação } \\
\text { Índice AW } \\
\text { (Citação } \\
\text { no } \\
\text { Tempo) } \\
\end{array}$ & Índice $\mathrm{g}$ & Índice e & $\begin{array}{l}\text { Assunçãc } \\
\text { da Iniciati }\end{array}$ & $\begin{array}{l}\text { explicita aos } \\
\text { a EQUATOR } \\
\text { de publicaçã }\end{array}$ & $\begin{array}{l}\text { ropósitos } \\
\text { is normas }\end{array}$ & $\begin{array}{c}\text { Internatio } \\
\text { nal } \\
\text { Committe } \\
\text { e of } \\
\text { Medical } \\
\text { Journal } \\
\text { Editors } \\
\end{array}$ \\
\hline $\begin{array}{l}\text { Revista Fisioterapia e Pesquisa (2316- } \\
\text { 9117) } \\
\text { Archives of Physical Medicine and }\end{array}$ & & 1 & 0 & 1,41 & 1 & 0 & Sim & Sim & Sim & Sim \\
\hline $\begin{array}{l}\text { Rehabilitation (0003-9993) } \\
\text { Eur. Journal of Obstetrics \& Gynecology }\end{array}$ & & 7 & 2 & 18,71 & 8 & 3,87 & Sim & Sim & Sim & Sim \\
\hline $\begin{array}{l}\text { and Reproductive Biology (0301-2115) } \\
\text { Muscles, Ligaments and Tendons }\end{array}$ & & 6 & 2 & 17,23 & 9 & 5,74 & Não & Sim & Não & Não \\
\hline $\begin{array}{l}\text { Journal }(2240-4554) \\
\text { Revista Brasileira de Geriatria e }\end{array}$ & & 1 & 1 & 2,83 & 1 & 1 & Sim & Sim & Sim & Sim \\
\hline Gerontologia (1981-2256) & & 1 & 0 & 1,41 & 1 & 0 & Sim & Sim & Sim & Sim \\
\hline Revista de Saúde Pública (1518-8787) & $\Delta 1$ & 2 & 1 & 6 & 2 & 1,41 & Sim & Sim & Sim & Sim \\
\hline European Journal of Pain (1532-2149) & & 7 & 2 & 16,52 & 8 & 4 & Sim & Sim & Sim & Não \\
\hline $\begin{array}{l}\text { American Journal of Physical Medicine \& } \\
\text { Rehabilitation (1537-7385) } \\
\text { Revista Brasileira de Medicina do }\end{array}$ & $\begin{array}{l}\text { a } \\
\text { B1 }\end{array}$ & 5 & 2 & 13,45 & 6 & 2,83 & Sim & Sim & Sim & Não \\
\hline $\begin{array}{l}\text { Esporte (1806-9940) } \\
\text { Brazilian Journal of Physical Therapy }\end{array}$ & & 2 & 1 & 2 & 2 & 0 & Sim & Sim & Sim & Sim \\
\hline $\begin{array}{l}(1809-9246) \\
\text { Jornal Brasileiro de Pneumologia (1806- }\end{array}$ & & 2 & 1 & 5,29 & 2 & 1,41 & Sim & Sim & Sim & Sim \\
\hline $\begin{array}{l}\text { 3756) } \\
\text { Revista Fisioterapia em Movimento }\end{array}$ & & 1 & 0 & 4,69 & 1 & 1 & Sim & Sim & Sim & Sim \\
\hline $\begin{array}{l}\text { (1980-5918) } \\
\text { Revista Saúde \& Transformação Social }\end{array}$ & & 10 & 5 & 23,73 & 14 & 7,94 & Não & Não & Não & Sim \\
\hline$(2178-7085)$ & & 0 & 0 & 0 & 0 & 0 & Não & Não & Não & Sim \\
\hline $\begin{array}{l}\text { The Spine Journal (1529-9430) } \\
\text { Autonomic Neuroscience: Basic and }\end{array}$ & & 6 & 1 & 16,46 & 6 & 1,41 & Sim & Sim & Sim & Sim \\
\hline Clinical (1566-0702) & & 5 & 3 & 11,27 & 5 & 1,41 & Não & Não & Não & Não \\
\hline Journal of Physiotherapy (1836-9553) & & 4 & 2 & 8,25 & 5 & 2,24 & Não & Sim & Não & Sim \\
\hline $\begin{array}{l}\text { Revista Brasileira de Queimaduras (1982- } \\
\text { 1883) }\end{array}$ & & 0 & 0 & 0 & 0 & 0 & Sim & Sim & Sim & Sim \\
\hline
\end{tabular}




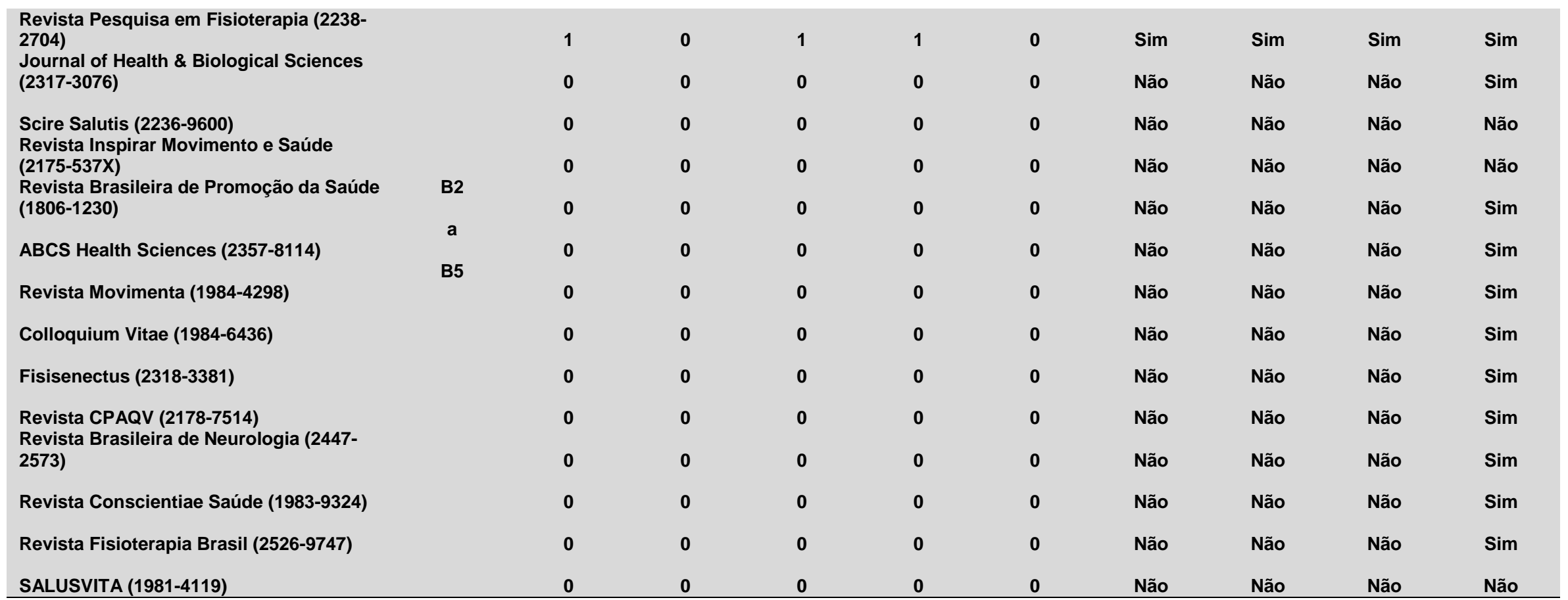




\section{Referências}

1. Barreto ML. O desafio de avaliar o impacto das ciências para além da bibliometria. Rev. Saúde Pública 2013;47(4):834-7.

2. Canadian Institute for Health Research: About knowledge translation; http:www.cihrirsc.gc.ca/e/29418.html.

3. Zidarov D, Thomas A, Poissant L. Knowledge translation in physical therapy: from theory to practice. Disabil Rehabil 2013. Early Online: 1-7.

4. LaRocca R, Yost J, Dobbins M, Ciliska M, Butt M. The effectiveness of knowledge translation strategies used in public health: a systematic review. BMC Public Health 2012;12:751.

5. Coury HJCG Vilella I. Perfil do pesquisador fisioterapeuta brasileiro. Rev Bras Fisioter 2009;13(4):356-63.

6. Silva TM, Costa LCM, Costa LOP. Evidence-Based Practice: a survey regarding behavior, knowledge, skills, resources, opinions and perceived barriers of Brazilian physical therapists from São Paulo state. Braz J Phys Ther 2015;19(4):294-303.

7. Silva TM, Costa LCM, Garcia NA, Costa LOP. What do physical therapists think about evidence-based practice? A systematic review. Man Ther. 2015;20(3):388-401.

8. Alshehri MA, Alalawi A, Alhasan H, Stokes E. Physiotherapists' behaviour, attitudes, awareness, knowledge and barriers in relation to evidencebased practice implementation in Saudi Arabia: a cross-sectional study. Int J Evid Based Healthc 2017;15:127-141.

9. Reis Filho AJS, Andrade BB, Mendonça VRR, Barral-Netto M. Conhecimento científico na graduação do Brasil: comparação entre estudantes de Medicina e Direito. Einstein 2010;8(3 Pt 1):273-80.

10. Reinders JJ, Kropmans TJ, Cohen-Schotanus J. Extracurricular research experience of medical students and their scientific output after graduation. Med Educ 2005;39(2):237.

11. Chan L, Heinemann AW. Editorial - Clinical Trial Registration: the time has come. American Journal of Occupational Therapy 2016;70(1).

12. Fraga-Maia H. "Salami Science" ou "Salami Slicing": uma reflexão sobre a produção científica. Revista Pesquisa em Fisioterapia 2017;7(1):3-5.

13. Moher D, Shamseer L, Clarke M, Ghersi D, Liberati A, Petticrew M, Shekelle P, Stewart LA; PRISMA-P GROUP. Preferred reporting items 
for systematic review and meta-analysis protocols (PRISMA-P) 2015 statement. Systematic Reviews 2015;4:1.

14. Liberati A, Altman DG, Tetzlaff J, Mulrow C, Gøtzsche PC, loannidis JP et al. The PRISMA statement for reporting systematic reviews and metaanalyses of studies that evaluate healthcare interventions: explanation and elaboration. BMJ 2009;339:b2700.

15. Moher D, Hopewell S, Schulz KF, Montori V, Gøtzsche PC, Devereaux PJ et al. CONSORT 2010 Explanation and Elaboration: updated guidelines for reporting parallel group randomised trials. BMJ 2010;340:c869.

16. Santos FC, Boggio EB, Souza CM, Rosa PV, Silva MF, Rosa LHT. A associação entre capacidade física e probabilidade de internação hospitalar em idosos que vivem na comunidade. Fisioter Pesqui 2017;24(3):238-244 .

17. Oliveira NT, Freitas SM, Fuhro FF, Luz MA Jr, Amorim CF, Cabral CM. Muscle activation during pilates exercises in participants with chronic non specific low back pain: a cross-sectional case-control study. Arch Phys Med Rehabil 2017;98(1):88-95.

18. Gonçalves RM, Ferreira LG, Assumpção MS, Schivinski CIS. Impact of sports activities on respiratory function and mechanics in children. Fisioter Mov 2017; 30(3):485-492.

19. Lima POP, Camelo PRP, Ferreira VMLM, Nascimento PJS, Bezerra MA, Almeida GPL et al. Evaluation of the isokinetic muscle function, postural control and plantar pressure distribution in capoeira players: a crosssectional study. Muscles, Ligaments and Tendons Journal 2017;7(3):498503.

20. Souza CF, Vieira MCA, Nascimento RA, Moreira MA, Câmara SMA, Maciel ACC. Relação entre força e massa muscular em mulheres de meia-idade e idosas: um estudo transversal. Rev. Bras. Geriatr. Gerontol 2017; 20(5):661-670.

21. Sousa KM, Oliveira WIF, Alves EA, Gama ZAS. Factors associated with access to physical rehabilitation for victims of traffic accidents. Rev Saude Publica 2017;51:54.

22. Rodrigues WCC, Pinheiro LB, Lima AT, Battisti L, Mota MAG, Costa MC et al. Perfil epidemiológico e clínico de pacientes com queimaduras atendidos pela fisioterapia na Universidade Estadual de Goiás. Rev Bras Queimaduras 2017;16(2):1-6.

23. Sanchez MO, Souza FCA, Nascimento EF, Sousa MFA, Nery ES, Alvarenga AVM. Hábito parafuncional e disfunção temporomandibular, uma questão de gênero? SALUSVITA 2017;36(3):667-675. 
24. Oliveira EN, Santos KT, Reis LA. Força de preensão manual como indicador de funcionalidade em idosos. Revista Pesquisa em Fisioterapia 2017;7(3):384-392.

25. Pereira KAS, Nunes SEA, Belfort MGS, Miranda RSA, Horas AD, Almeida JP Júnior et al. Fatores de risco e proteção contra doenças crônicas não transmissíveis entre adolescentes. Rev Bras Promoç Saúde 2017;30(2):205-212.

26. Pereira TRC, Montesano FT, Ferreira PD, Minozzi AS, Beleza ACS. Existe associação entre os desconfortos no puerpério imediato e a via de parto? Um estudo observacional. ABCS Health Sci 2017;42(2):80-84.

27. Barbosa AM, Santos KM, Santana GN, Inocêncio SKM. Prevalência de lombalgia em fisioterapeutas estudantes de pós-graduação em Goiânia: um estudo transversal. Movimenta 2017;10(1):10-20.

28. Dias JM, Cisneros L, Dias R, Fritsch C, Gomes W, Pereira L et al. Hydrotherapy improves pain and function in older women with knee oste oarthritis: a randomized controlled trial. Braz J Phys Ther 2017;21(6):449456.

29. Gadelha CF, Revoredo AA, Pereira TJCC, Souza Pinheiro PI, Barbosa GM, Almeida LCA et al. Efeito do aquecimento sobre a flexibilidade e o desempenho funcional: ensaio clínico randomizado. Rev Bras Med Esporte 2017;23(5):385-389.

30. Rodrigues TA, Goroso DG, Westgate P, Carrico C, Batistella L R, Sawaki L. Slow versus fast robot-assisted locomotor training after severe stroke: a randomized controlled trial. Am J Phys Med Rehabil 2017;96(Suppl):S165-S170.

31. Gondim ITG, Lins CCSA, Asano NMJ, Asano AGC, Cabral ED, Coriolano MGWS. Individualized guidance and telephone monitoring in a selfsupervised home-based physiotherapeutic program in Parkinson. Fisioter Mov 2017;30(3): 559-568.

32. Machado AS, Pires-Neto RC, Carvalho MTX, Soares JC, Cardoso DM, Albuquerque IM. Efeito do exercício passivo em cicloergômetro na força muscular, tempo de ventilação mecânica e internação hospitalar em pacientes críticos: ensaio clínico randomizado. J Bras Pneumol 2017;43(2):134-139.

33. Hazime FA, Baptista AF, Freitas DG, Monteiro RL, Maretto RH, Hassue SMA, João SMA. Treating low back pain with combined cerebral and peripheral electrical stimulation: a randomized, double-blind, factorial clinical trial. Eur J Pain 2017;21:1132-1143. 
34. Sachetti A, Dal'Acqua AM, Lemos FA, Naue WS, Laura JS, Bianchi T et al. Efeitos da estimulação elétrica neuromuscular sobre a mobilidade diafragmática de pacientes críticos: ensaio clínico randomizado. ConScientiae Saúde 2017 16(2):224-233.

35. Sousa ASK, Lopes KL, Fragnani SG, Nery T, Werner E, Bezerra PP. Fisioterapia associada à yoga e Musicoterapia na doença de Parkinson: Ensaio clínico. Rev Bras Neurol 2017;53(3):31-40.

36. Carneiro CDL, Gonçalves ACB, Gomes CAC, Ferreira FS, Rocha RSB Torres $\mathrm{DC}$ et al. Análise dos parâmetros ventilométricos como resposta a estimulação diafragmática elétrica transcutânea em pacientes sob suporte ventilatório: um ensaio clínico randomizado. Revista CPAQV 2017;9(3):1-11.

37. Belmonte LM, Gerent PM, Silva FZ, Lima IAX, Belmonte LAO. Efeito do exercício aquático terapêutico em mulheres com osteoartrose de joelho: um estudo randomizado controlado. FisiSenectus 2017;5(1):31-41.

38. Silva HF, Castrillón CIM, Figueiredo MPF, Vanderlei FM, Linares SN, Lemos LK et al. Comportamento da pressão arterial e composição corporal após dois tipos de treinamentos periodizados em participantes com síndrome metabólica. Ensaio clínico controlado randomizado. Colloq Vitae 2017;9(1):28-33.

39. Oliveira MC, Ortiz Sobrinho C, Orsini M. Comparação entre o método Reequilíbrio Toracoabdominal e a fisioterapia respiratória convencional em recém-nascidos com taquipneia transitória: um ensaio clínico randomizado. Fisioterapia Brasil 2017;18(5);598-607.

40. Abreu RM, Rehder-Santos P, Minatel V, Dos Santos GL, Catai AM. Effects of inspiratory muscle training on cardiovascular autonomic control : A systematic review. Auton Neurosci 2017;208:29-35.

41. Dario AB, Moreti Cabral A, Almeida L, Ferreira ML, Refshauge K, Simic M et al. Effectiveness of telehealth-based interventions in the management of non-specific low back pain: a systematic review with meta-analysis. Spine J 2017;17(9):1342-1351.

42. Wolpe RE, Zomkowski K, Silva FP, Queiroz APA, Sperandio FF. Prevalence of female sexual dysfunction in Brazil: a systematic review. European Journal of Obstetrics \& Gynecology and Reproductive Biology 2017; 211: 26-32.

43. Zanelat CF, Rocha FR, Lopes GM, Ferreira JR, Gabriel LS, Oliveira TG. The respiratory physiotherapy causes pain in newborns? A systematic review. Fisioter Mov 2017; 30(1):177-86.

44. Medeiros AIC, Fuzari HKB, Rattes C, Brandão DC, de Melo Marinho PE. Inspiratory muscle training improves respiratory muscle strength, 
functional capacity and quality of life in patients with chronic kidney disease: a systematic review. Journal of Physiotherapy 2017;63:76-83.

45. Almeida KM, Fonseca ST, Figueiredo PRP, Aquino AA, Mancini MC. Effects of interventions with therapeutic suits (clothing)

on impairments and functional limitations of children with cerebral palsy: a systematic review. Braz J Phys Ther 2017;21(5):307-320.

46. Santos CLC, Sobrinho CLN, Barbosa GB. Síndrome de Burnout em fisioterapeutas: uma revisão sistemática. Revista Pesquisa em Fisioterapia 2017;7(1):103-114.

47. Cordeiro AL, Lima TG. Fisioterapia em unidades de emergência: uma revisão sistemática. Revista Pesquisa em Fisioterapia 2017;7(2):276-281.

48. Silva IC, Campos NG, Vinhote JFC, Florêncio ACL, Marizeiro DF, Braga DK et al. Atuação da fisioterapia em pacientes transplantados de medula óssea: revisão sistemática de literature. J Health Biol Sci 2017;5(4):371377.

49. Jorge MSG, Knob B, Ribeiro DS, Zanin C, Wibelinger LM. Efeitos da reabilitação fisioterapêutica nas mãos de indivíduos com doenças reumáticas: revisão sistemática. REVISTA INSPIRAR Movimento \& Saúde 2017;14(3):39-47.

50. Garção DC, Sobral JM, Santos JCD, Mendonça VBD, Rezende LS, Santana MMS et al. Intervenção fisioterapêutica com suporte parcial de peso em indivíduos pós-ave: revisão sistemática. Scire Salutis 2017;7(1):6-14.

51. Santos BW. Brinquedo terapêutico na fisioterapia respiratória em pediatria: Uma revisão sistemática. Saúde \& Transformação Social 2017;8(2):120-127. 